\title{
Numerical simulation of elastic wave propagation in masonry compared with acoustic emission experimental results
}

\section{G. Livitsanos, N. Shetty, E. Verstrynge, M. Wevers, D. Van Hemelrijck \& D. G. Aggelis}

Archives of Civil and Mechanical Engineering

e-ISSN 1644-9665

Volume 20

Number 1

Archiv.Civ.Mech.Eng (2020) 20:1-11

DOI 10.1007/s43452-020-00019-z 
Your article is protected by copyright and all rights are held exclusively by Wroclaw University of Science and Technology. This eoffprint is for personal use only and shall not be self-archived in electronic repositories. If you wish to self-archive your article, please use the accepted manuscript version for posting on your own website. You may further deposit the accepted manuscript version in any repository, provided it is only made publicly available 12 months after official publication or later and provided acknowledgement is given to the original source of publication and a link is inserted to the published article on Springer's website. The link must be accompanied by the following text: "The final publication is available at link.springer.com". 


\title{
Numerical simulation of elastic wave propagation in masonry compared with acoustic emission experimental results
}

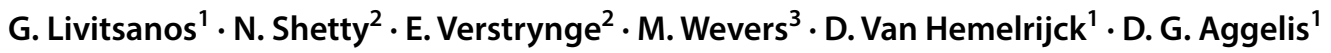

Received: 8 March 2019 / Accepted: 13 December 2019

(c) Wroclaw University of Science and Technology 2020

\begin{abstract}
It has been shown in acoustic emission literature that the distance between a possible cracking event and a receiver is affecting the signal parameters providing a wrong image of the real fracture characteristics. In this study, lab-scale experimental tests on masonry components are performed in order to determine the disturbance of the acoustic emission wave properties and verify the experimental observations via numerical wave propagation analysis. The investigation is extended to different geometries including "couplets," "triplets" and masonry walls. The simulations allow to understand, verify and predict the acoustic emission signal properties alterations in many different types of masonry experiments as well as the correct characterization of the fracture mode.
\end{abstract}

Keywords Masonry $\cdot$ Heterogeneity $\cdot$ Acoustic emission $\cdot$ Nondestructive testing $\cdot$ Elastic wave propagation $\cdot$ Simulation

\section{Introduction}

Historic buildings constructed on the basis of stone or brick masonries require dedicated monitoring. Since many of these structures are of high cultural heritage importance, nondestructive evaluation (NDE) is the standard approach for structural integrity evaluation and life assessment prediction. One of the main NDE techniques, widely known and applied in many different material fields, is the acoustic emission (AE) technique, which is capable of detection and analysis of the elastic waves produced by the failure mechanisms [1, 2]. It has been shown that different cracking modes can be accurately classified by $\mathrm{AE}$, and as a result, this technique can be used as pre-failure warning [3, 4]. This is due to the fact that the shape of the waveforms has proven indicative of the fracture type. For example, it has been shown that shear events are characterized by longer "rise time" than tensile events [5] meaning essentially that

G. Livitsanos

georgios.livitsanos@vub.be

1 Department Mechanics of Materials and Constructions (MEMC), Vrije Universiteit Brussel, Brussels, Belgium

2 Building Materials and Building Technology Division, Civil Engineering Department, KU Leuven, Leuven, Belgium

3 Department of Materials Engineering, KU Leuven, Leuven, Belgium
AE signals from shear events have longer duration before they reach their peak amplitude. Another parameter that has been used for the characterization of the cracking mode is the average frequency. A shift from higher to lower values indicates a shift of the cracking mode from tensile to shear [6]. In several structural materials like concrete or stone, characterization of fracture mode is possible through specific AE parameters [7-11]. In masonry only recently this approach has been tested proving quite promising. Specifically, in couplet specimens cracks that occurred under shear strain, as confirmed by digital image correlation technique, exhibited lower frequencies and longer durations than $\mathrm{AE}$ signals originating from tensile cracks [12]. However, due to the well-known heterogeneity of masonry, these correlations cannot be directly taken for granted for different or larger geometries. Recent studies proved also that the $\mathrm{AE}$ signals undergo strong changes due to the propagation distance from the source to the receivers in different material fields [13]. This practically means that one specific AE event will be recorded as having different waveform shapes for sensors placed at close or further distances from the source. Furthermore, since the shape of the wave changes, the value of AE parameters is expected to be affected as well. If this is not accounted for, the risk of erroneous characterization increases, especially for large-scale components where attenuation and scattering accumulate over longer distances. Therefore, the influence of the distance in the measurement 
of AE parameters should be studied, especially in relation to standardization. This is currently being attempted for the field of concrete [14]. However, masonry components and structures present higher heterogeneity. The different material properties, the presence of the interfaces, as well as heterogeneities such as cracks and voids hinder the accuracy of AE-based classification as they complicate the scattering conditions during wave propagation, while damping additionally contributes to the distortion of the signals. Therefore, the aim of this paper is to examine the effect of wave propagation in masonry components in order to establish a robust relation between propagation characteristics and material properties. In this investigation, numerical simulations are addressed to examine wave propagation and specifically the change in the waveform shape and parameters and to provide a comparison with the respective experimental results. Numerical simulations of wave propagation focusing on AE signals have been conducted for different materials $[15,16]$. The example of composites is characteristic where apart from the heterogeneity, there is a strong amount of dispersion due to Lamb wave dispersion [17, 18], while the situation in metal plates is similar [15]. In any case, the change in $\mathrm{AE}$ waveforms and parameters is apparent, mainly due to the plate wave dispersion that tends to separate the different wave modes after long propagation. Separation of modes is also seen in concrete after a simulated excitation of a surface crack and is attributed to the different wave velocities of longitudinal, shear and Rayleigh wave modes. The degree of heterogeneity (e.g., in the form of cracks) intensifies this phenomenon due to scattering [13]. In masonry, the repeated pattern of geometry between bricks and mortar is expected to induce also a great amount of scattering seriously altering the waveform characteristics. An earlier work referring to masonry studied the spreading of an ultrasonic beam [19] without, however, focusing on the exact waveform shape, which is the focus of the present study. Simulations offer good understanding of the mechanics of propagation (including different modes, reflection, correlation to mechanical properties and damage existence), and it is the first time that they are used to explain the waveform distortion of AE signals in masonry. Simulations in this case can help distinguishing up to what extent a change in $\mathrm{AE}$ waveform shape is due to corresponding changes in fracture mode or due to the propagation distance.

In this paper, numerical simulations are used in order to produce elastic wave propagation results for various cases of masonry layout. The specific cases discussed herein concern the comparison between AE parameters obtained in lab-scale masonry experiments and via numerical wave simulation on the same geometries. The geometries that are being investigated are three different lab-scale masonry components, namely couplets, triplets and walls, and it is revealed that even in small scale the influence of the propagation distance should not be neglected in AE-based failure mode characterization. Starting with the experimental details in Sect. 2, the materials that are being used and the different masonry geometries that are being investigated are described. In Sect. 3, the basic principle of the wave equation that is being used in the numerical approach as well as all the parameters that are predefined for the accurate simulations of all the masonry models is described. Finally, Sect. 4 presents the experimental and numerical AE parameters (RT and AF) evolution in terms of the distance, while effective correlations between them signify the importance of this study. The main conclusions are finally presented in Sect. 5.

\section{Experimental details}

\subsection{Materials and setup}

Red clay bricks (RCB) with a density of $1653 \mathrm{~kg} / \mathrm{m}^{3}$ and dimensions $188 \times 88 \times 63 \mathrm{~mm}$ were used. The mortar is a lime-cement (LC) mortar (Table 1). Before applying the mortar layers, the bricks were submerged in water for 2 min to avoid excessive absorption of the mortar's water into the pores of the bricks. Firstly, masonry couplets (Fig. 1a) were composed. The connection of the bricks on top of each other included one mortar layer in between with thickness of approximately $12 \mathrm{~mm}$. Two more mortar layers were added, on top and on the bottom of the specimen in order to contribute also in the triaxial stress state and provide a better representation of a small part of a masonry wall specimen. Finally, the overall dimension after the assemblance is of $110 \mathrm{~mm}$ height and of $194 \mathrm{~mm}$ length (Fig. 1a). These specimens were tested under uniaxial compression in order to investigate the mortar-brick interface behavior. Similar procedure was also followed in the triplets. The arrangement of bricks in a triplet specimen consists of three bricks connected next to each other by two mortar layers (Fig. 1b). In this specific case, the middle brick was placed in a way that provides the two thirds of the total plane surface as a contact plane for the shear forces undertakes (Fig. 1b), as has been commonly applied in the literature [20-22]. In this

Table 1 Composition of mortar, similar to [26]

\begin{tabular}{ll}
\hline Composition & Lime-cement (LC) mortar \\
\hline River sand $(\mathrm{g})$ & 2700 \\
Binder $(\mathrm{g})$ & 572.5 \\
& $66.7 \%$ CL90S (EN 459-1:2001) \\
& $33.3 \%$ CEM I 42.5 R \\
Water at $20^{\circ} \mathrm{C}(\mathrm{g})$ & 583.3 \\
Volume density $\left(\mathrm{kg} / \mathrm{m}^{3}\right)$ & 1758 \\
\hline
\end{tabular}



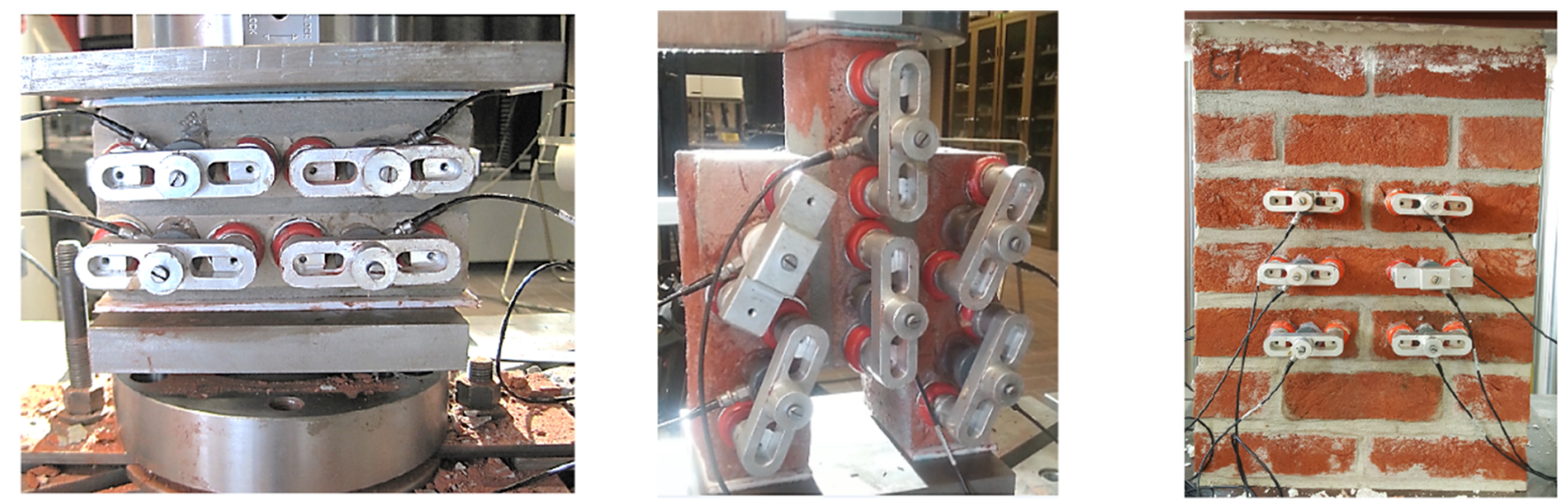

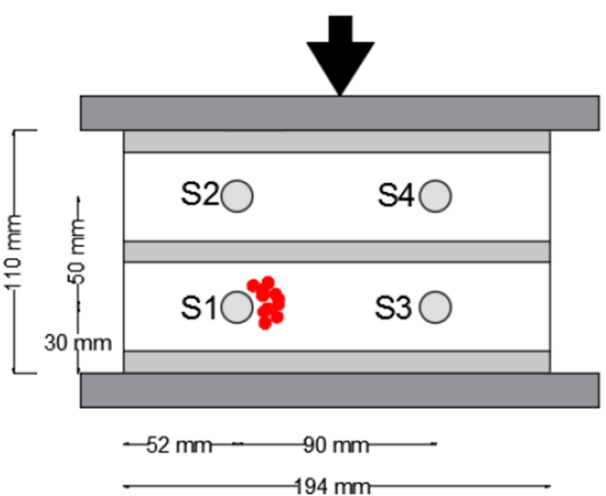

(a)

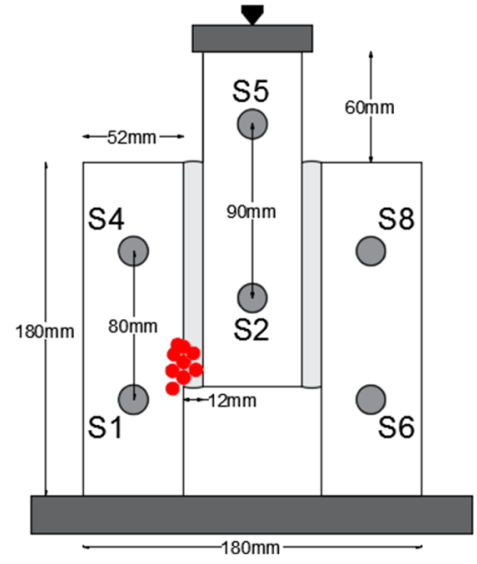

(b)

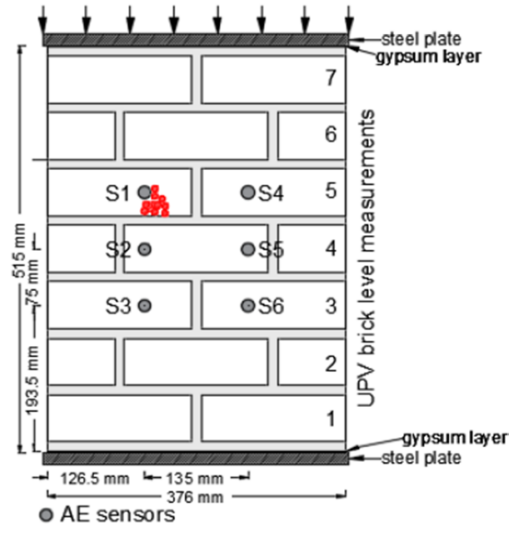

(c)

Fig. 1 Experimental wave propagation setup of a masonry couplet, $\mathbf{b}$ masonry triplet, $\mathbf{c}$ masonry wall. Event selection from a fractured area close to sensor 1 (S1) indicated by red dots

specific case, zero normal horizontal stress was applied [23-25]. This masonry element setup was selected in order to provoke shear in the mortar-brick interface under static monotonic loading. During the displacement-controlled testing of both couplets and triplets, a monotonic load was applied until the final failure using an Instron 5885 testing machine with a $250 \mathrm{KN}$ capacity load cell with a rate $0.5 \mathrm{~mm} / \mathrm{min}$ and $0.2 \mathrm{~mm} / \mathrm{min}$, respectively. In order to upscale the experimental investigation, compression tests on masonry wall specimens were conducted with dimensions of $515 \times 376 \times 88.5 \mathrm{~mm}^{3}$. Figure $1 \mathrm{c}$ shows the details and dimensions of the masonry wall. A typical bond layout was selected to simulate more realistic conditions. They were subjected to cyclic incremental compression tests with a specific steady step for all of the cycles using a $2500 \mathrm{kN}$ universal testing machine "Instron" at $0.25 \mathrm{kN} / \mathrm{s}$ loading rate under force control. In every case, a thin layer of gypsum and a plastic sheet were placed on the top and bottom of the wall and slightly pressed under the platens of the mechanical press to make it a flat surface for an equal distribution of the load.

\subsection{AE testing}

During testing of all the masonry geometries, acoustic emission (AE) monitoring took place by means of piezoelectric transducers. The sensors that were used are the R15-type sensors $(150 \mathrm{kHz}$ resonance frequency with $40 \mathrm{~dB}$ preamplifier). The computer is equipped by PCI/DSP-4 Data Acquisition Boards with eight channel inputs with sampling rate up to $10 \mathrm{MHz}$. The threshold was $35 \mathrm{~dB}$ [27]. Acoustic coupling was improved by Vaseline (petroleum jelly) between the sensors face and the specimens' surface. The piezoelectric sensors were placed on the same side of each specimen ( Fig. 1) by the use of metallic clamps to assure a proper attachment until the final failure of the specimens. The waveforms collected by the sensors are treated in terms of their basic parameters. For accurate AE data collection and source localization, two different planar AE localization approaches were assumed. In the first case, homogeneous velocity was assumed for each material and each masonry component, while in the second case, different velocities in two orthogonal directions were used as an input. However, 
this technique requires a priori knowledge of the directiondependent velocity profile and as a result the materials' properties.

In the case of couplets, the AE activity was acquired by applying planar AE localization by four sensors where two sensors were placed on each of the two bricks on either sides of the central mortar joint (Fig. 1a). In the case of triplets, two sensors were placed on each of the three bricks, resulting in a setup with six AE sensors (Fig. 1b). In the case of the masonry wall component, a suitable layout with six $\mathrm{AE}$ sensors was selected as shown in Fig. 1. For the optimum position of them, the AE sensors were placed far apart, and pulse calibration was performed. AE hit arrival time and amplitude were checked to witness the effect of distance and attenuation on the signal acquisition and localization accuracy. If the AE detection was not accurate, the distance between the sensors was decreased. In this manner, a suitable layout of AE sensors was selected for the tests in the masonry wall specimens.

During the tests, AE hits were recorded upon exceedance of the threshold and subsequently assembled into AE events. Some of the main recorded AE signal features are the maximum amplitude, A (usually in $\mathrm{dB}$ ), and the rise time, RT (in $\mu \mathrm{s})$, which is the time between the first threshold crossing and the point of peak amplitude. Frequency content can be measured by average frequency, $\mathrm{AF}$ (in $\mathrm{KHz}$ ), which is the total number of threshold crossings divided by the duration [28]. AE signals being recorded reasonably close in time to each other were classified in "AE events" and localized through the arrival delay at the different sensors.

In all of the aforementioned different cases, a number of ten localized events are shown with red dots in the specimens and they represent any signal source that corresponds to a possible crack initiation (Fig. 1). These events were selected in a way that their values are close to the average ones resulting from the two-dimensional planar orthotropic localization close to sensor 1 (S1). Choosing an event close to a specific sensor allows to investigate how the AE parameters of this event are received by different sensors at different distances. The AE events received from couplets were considered indicative of tensile splitting fracture, while events from triplets were indicative of tensile and shear failure.

The nature of the selected events for the cases of couplet and triplet specimens was confirmed by the strain fields obtained by DIC as it can be seen in Figs. 2 and 3. In each case, the observation of the DIC surface strain evolutions next to sensor 1 was deemed necessary for the AE event selections from the same areas of interest and at the same time intervals. For instance, in the case of couplet (Fig. 2) at $81 \%$ of the load there is high lateral $\varepsilon_{\mathrm{xx}}$ strain evolution close to sensor 1 (S1) (values vary between 0.014 and 0.0246) (Fig. 2a), while for the same load percentage, the shear $\varepsilon_{\mathrm{xy}}$ strain evolution varies between -0.003 and -0.0051 (Fig. 2b).

Moreover, in the case of the triplet specimen (Fig. 3) where shear-dominated fracture in the mortar layer was investigated, at $74 \%$ of the load there was shear $\varepsilon_{x y}$ strain concentration in the area indicated by the oval shape where strain values varied from -0.00093 to -0.0017 (Fig. 3a). At the same load percentage, lateral $\varepsilon_{x x}$ strain values varied between 0.00042 and 0.00008 signifying the higher shear proportion (Fig. 3b).

Investigating how the $\mathrm{AE}$ parameters of a specific event are received by different sensors in different distances allows the understanding of the influence of propagation, while it is a suitable data set for comparison with the simulation results.

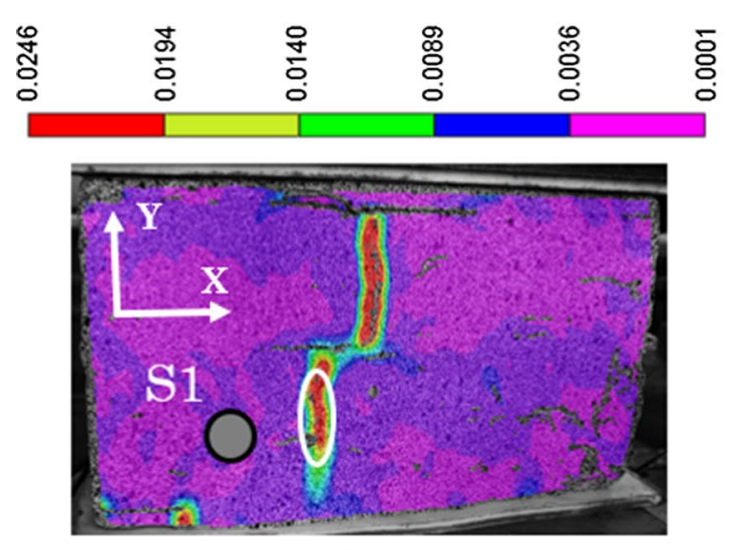

(a)

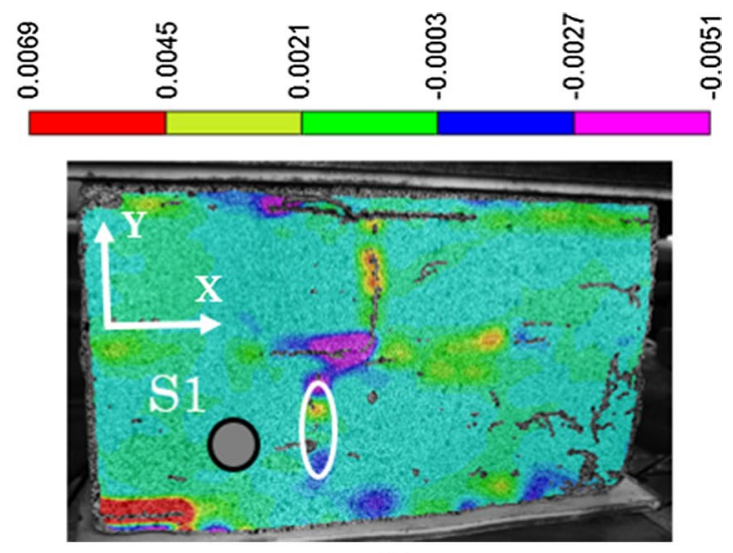

(b)

Fig. 2 Full field strain map of a lateral $\varepsilon_{x x}$ strain and $\mathbf{b}$ shear $\varepsilon_{x y}$ strain on masonry couplet at $81 \%$ of load. Event selection from a fractured area close to sensor 1 ( $\mathrm{S} 1$ - gray circle) indicated by oval shapes 


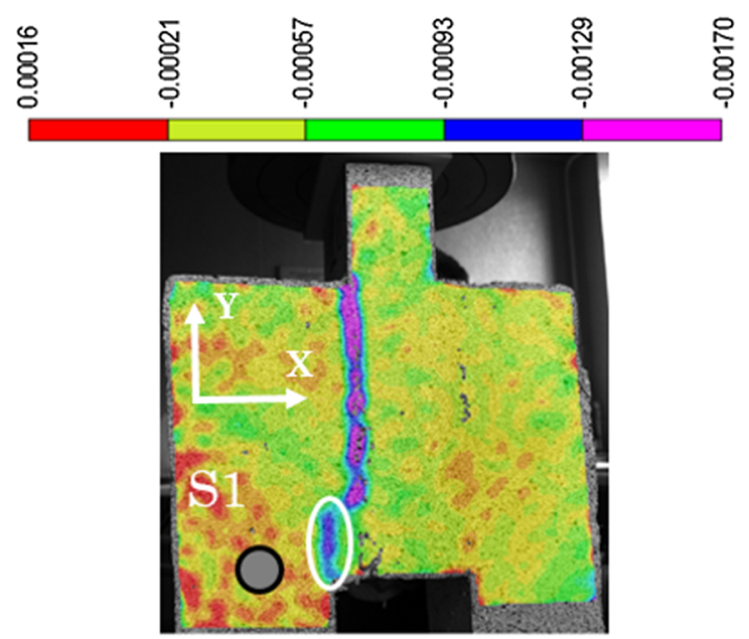

(a)

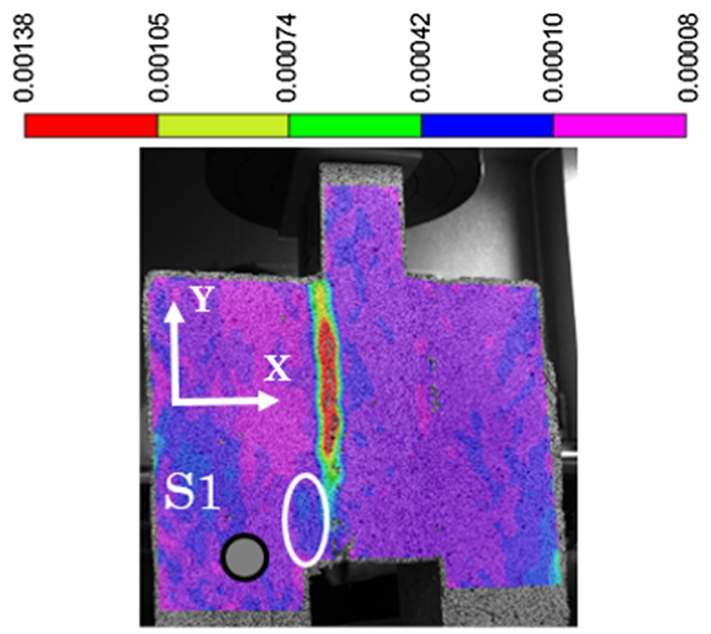

(b)

Fig. 3 Full field strain map of a shear $\varepsilon_{x y}$ strain and $\mathbf{b}$ lateral $\varepsilon_{x x}$ strain, on masonry triplet at $74 \%$ of load. Event selection from a fractured area close to sensor 1 ( $\mathrm{S} 1$ - gray circle) indicated by oval shapes

Table 2 Material properties of the wave propagation simulation

\begin{tabular}{llllc}
\hline & $\rho\left(\mathrm{kg} / \mathrm{m}^{3}\right)$ & $C_{\mathrm{p}}(\mathrm{m} / \mathrm{s})$ & $\lambda(\mathrm{MPa})$ & $\mu(\mathrm{MPa})$ \\
\hline Brick & 1653 & 1440 & 660 & 1282 \\
LC mortar & 1758 & 1000 & 510 & 531 \\
\hline
\end{tabular}

\section{Numerical wave propagation simulation}

Numerical simulation of wave propagation was studied for the same layout of masonry specimens. AE parameters such as RT and AF were calculated to investigate the influence of the distance pulser-receiver. The numerical simulations were conducted by a specialized commercially available software, Wave2000 [29]. The principle is based on the solution of the wave Eq. (1) using the finite difference method in the plane strain case with respect to the boundary conditions of the model, which includes the input source that has predefined time-dependent displacements at a given location and a set of initial conditions:

$\rho \frac{\theta^{2} u}{\theta t^{2}}=\left(\mu+\eta \frac{\theta}{\theta t}\right) \nabla^{2} u+\left(\lambda+\mu+\varphi \frac{\theta}{\theta t}+\frac{n}{3} \frac{\theta}{\theta t}\right) \nabla(\nabla \cdot u)$

This equation is expressed by $u(\mathrm{~m})$ being the time-varying displacement vector which consist of two vertical components, $u_{x}$ and $u_{y}$, the density $\rho\left(\mathrm{kg} / \mathrm{m}^{3}\right)$, the first $\lambda$ and the second $\mu$ Lame constants (Pa), the "shear" $\eta$ and the "bulk" $\varphi$ viscosities (Pa s) and the time $t$ (s). The calibration of the numerical model is based on the brick and mortar properties, as experimentally measured (Table 2). The first and the second lame constants are related to the Young's modulus and the Poisson ratio. These parameters were obtained

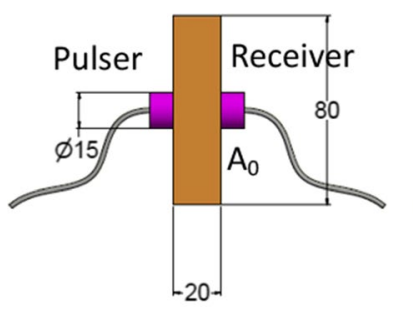

(a)

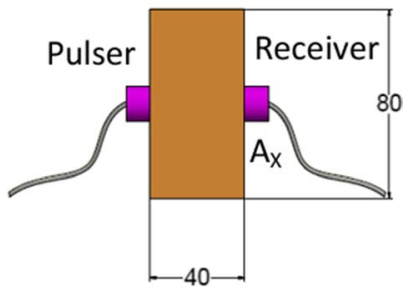

(b)
Fig. 4 Experimental setup of the attenuation measurements on brick material, a half brick specimen $(20 \mathrm{~mm})$, b full brick specimen $(40 \mathrm{~mm})$

experimentally from compressive tests, calculated from the surface strains that were monitored using the digital image correlation (DIC) technique.

Attenuation was experimentally measured by comparing the amplitudes received after wave propagation through a normal brick thickness and its half (Fig. 4), keeping a constant excitation. The attenuation coefficient was determined by measuring the reduction in amplitude of an ultrasonic wave, which had traveled a known distance through a material and is given by:

$\alpha=-\frac{20}{x} \log \left(\frac{A_{x}}{A_{0}}\right)$

where $A_{0}$ is the initial peak amplitude of the wave and $A_{x}$ is the peak amplitude after a travel distance $x$ (Fig. 4).

This resulted in an average value of $1.67 \mathrm{~dB} / \mathrm{cm}$ for the RCB material. Therefore, the values of $n$ and $\varphi$ in Eq. 2 were adjusted so that the resulting attenuation is similar to the experimental result. 
For the simulations, the imposed displacement signal excitation consists of a sine enveloped waveform of five cycles, running the simulation under the $150 \mathrm{kHz}$ frequency. In all the simulation cases, the length of the excitation was $10 \mathrm{~mm}$ so as to accurately simulate the length of the event selection area. The receivers provided the average vertical displacement over their length, meaning that the receiver signal represented the average response over a number of nodes. The numerical models and indicative snapshots of the strain field in the different masonry geometries are depicted in Fig. 5.

\subsection{Convergence}

In order for the simulation to produce reliable results, it is essential that certain guidelines are followed concerning mainly the spacing and time resolution of the wave equation solution. One important rule of thumb implies the mesh size to be one tenth (or lower) than the major wave length, while in the time domain, the sampling rate should be at least ten times higher than the major frequency [30]. Consequently, the optimum spacing resolution was investigated in order to minimize the calculating error, while the simulations could run in a timely manner. In terms of size, the couplet specimen is the smallest in comparison with the triplets and the walls. The selected resolution for this specimen is applied for all specimens in order to have a valid comparison. Transit time was monitored in the couplet specimen, between sensor 1 and sensor 3, applying different spacing resolutions starting from 10 down to $1 \mathrm{~mm}$ (Fig. 6). Finer mesh was not practical as it required several hours of PC time $(3.20 \mathrm{GHz}$, 4 CPUs, 8192-MB RAM).

The transit time seems to converge approaching $1 \mathrm{~mm}$, and therefore, the value of $1.3 \mathrm{~mm}$ was selected which

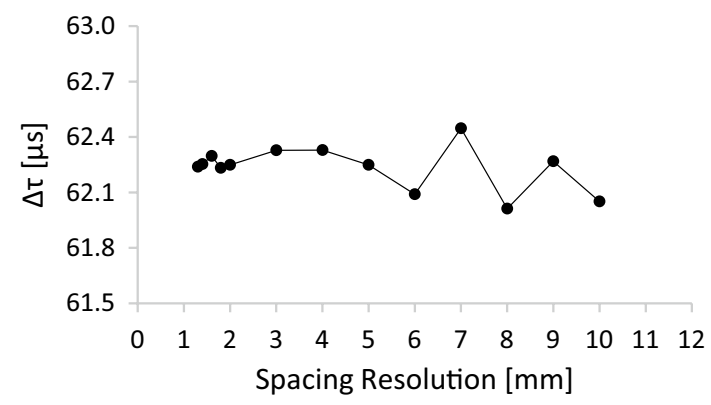

Fig. 6 Spacing resolution in terms of signal arrival time between two sensors

presents a difference of only $0.095 \%$ to the result of $1 \mathrm{~mm}$, and at the same time, it consumed only 12 min of calculation time. This value is very close to one tenth of the excited wavelength (approximately $10 \mathrm{~mm}$ for frequency of $150 \mathrm{kHz}$ ). The sampling time was $0.197 \mu$ s much less than the period of the excited wave $(7 \mu \mathrm{s})$, enabling each cycle to be represented by approximately 36 points, while $10-20$ points are considered satisfactory [30].

\section{Results}

\subsection{Experimental: numerical propagation distance effect on AE parameters}

Before presenting the numerical result, the behavior of $\mathrm{AE}$ waves as measured experimentally is illustrated to form the basis for discussion. As aforementioned, this involves ten events localized close to one of the AE sensors (S1). This way the wave received at $\mathrm{S} 1$ is assumed as reference and in

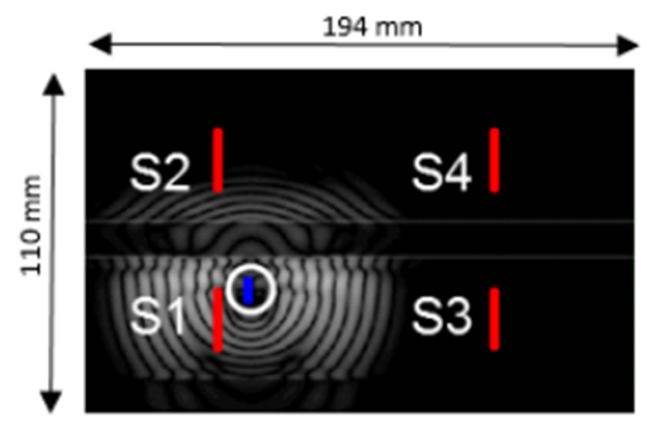

(a)

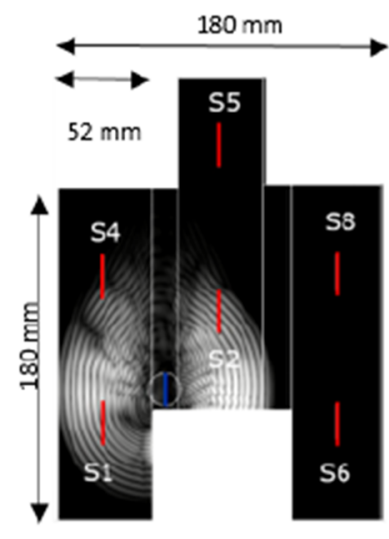

(b)

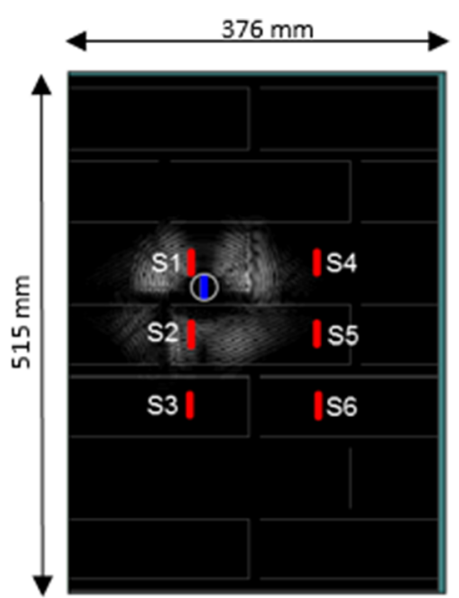

(c)

Fig. 5 Numerical wave propagation setup of a masonry couplet, b masonry triplet, c masonry wall. Simulation of similar excitation with the event selection area in Fig. 1. The source is the blue line in the white circle 
general free from distortion enabling the comparison with the parameters of the other sensors at longer distances. Figure 7 presents the evolution of RT and AF, as recorded by all sensors applied on the different specimens. Each dot represents the average from all the waveforms of the selected events, namely ten different AE signals.

For the case of couplet (Fig. 7a), the RT of the waveform exhibits a steadily increasing trend with propagation distance. Specifically, sensor 4 presents the highest value as this is the most remote one. While RT is registered with an average value of $60 \mu$ s at sensor 1 , it monotonically increases reaching $292 \mu$ s at sensor 4 at a distance of $106 \mathrm{~mm}$. On the other hand, and despite the use of resonant sensors, AF content decreases from 71 to $55 \mathrm{kHz}$, as depicted in Fig. 7a bottom.

A similar trend is followed in the case of masonry triplet where two different signal excitations were identified. In this experiment, shear failure was dominating in the mortar joint, and thus, the AE sources were primarily identified as shear events. However, it was observed that due to the lack of lateral confinement, horizontal movement of the edge bricks was possible. Consequently, the fracture was a combination of tensile (Mode I) and shear (Mode II) failure of the mortar joint triggering individual $\mathrm{AE}$ events with respective characteristics closer to one or the other mode [22, 31]. To determine the excitation type experimentally, the aid of DIC was crucial, as it indicated the different strain components according to the time of occurrence. The accurate correlation of these events with the acoustic emission provided the possibility to distinguish the different types of events in the different time intervals.

Concerning the experimental excitation Mode II (shear), RT starts at $153 \mu$ s in S1 and reaches $274 \mu$ s in the most remote sensor (S5-152.3 mm). Mode I excitation follows a similar increasing trend, starting naturally from lower RT value, which is typical for tensile events compared to shear ones $[5,8,9,14,15]$. This has been attributed to the larger proportion of longitudinal elastic waves which arrive earlier for tensile than for shear modes, causing the peak at earlier time within the waveform, resulting thus in shorter rise time. In this case, the shift is even stronger starting at $39 \mu \mathrm{s}$ for the tensile mode, before reaching $254 \mu$ s for the longest distance. Furthermore, we can observe that RT for tensile excitation at S5 sensor is larger than RT for shear excitation at $\mathrm{S} 1$ sensor. This indicates the influence of propagation distance and the importance of applying localization, in order to attempt accurate fracture characterization. Moreover, the average differences between the different modes are quite strong for the nearest sensor S1, but these differences diminish with distance, and at S5 and S8, tensile and shear signals look practically the same. Specifically, for sensor $\mathrm{S} 1$ close to the source, the difference in RT is quite high (114 $\mu$ s between shear and tension), while for a distance of $78 \mathrm{~mm}$ from the source (S2), the difference is still large but has already dropped to $79 \mu \mathrm{s}$. As for the AF, there is again a decreasing trend with distance, which is more obvious for the tensile signals.
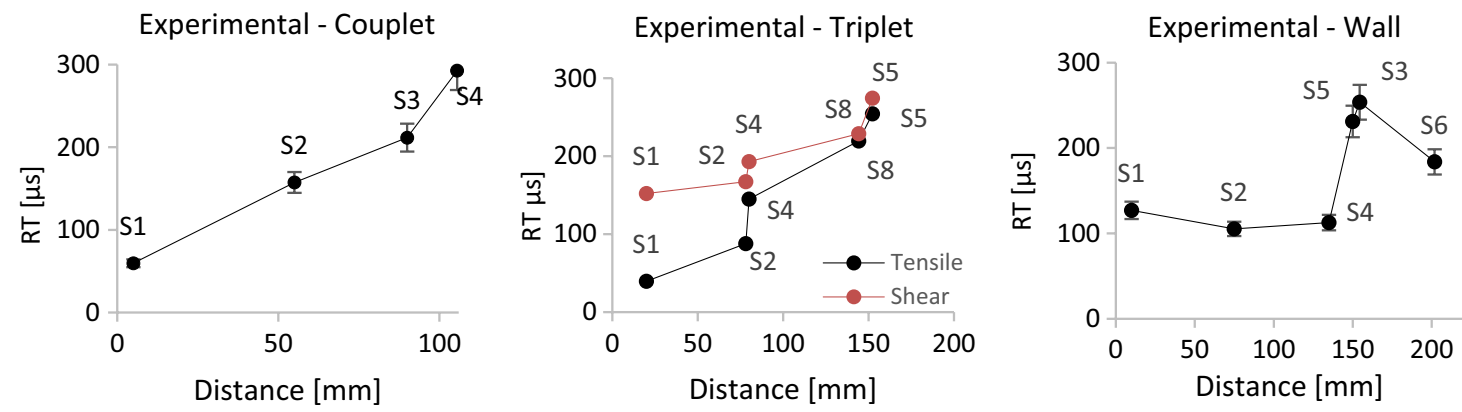

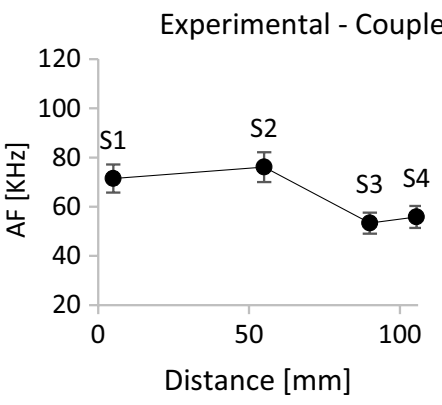

(a)

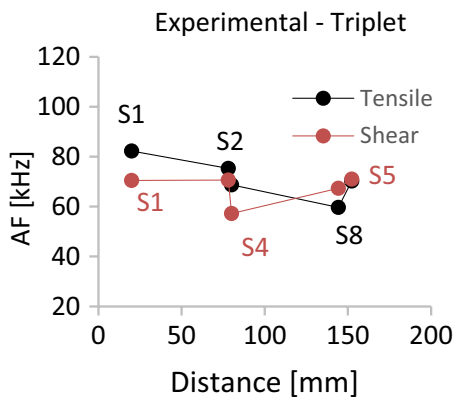

(b)

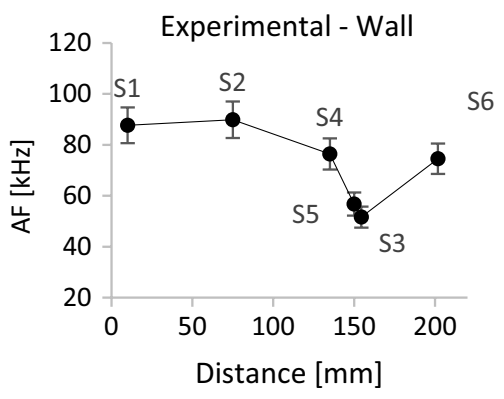

(c)

Fig. 7 Experimental propagation distance effect in RT and AF in a masonry couplets, $\mathbf{b}$ masonry triplets, $\mathbf{c}$ masonry wall specimens 
It is interesting to mention that sensor 6 did not register any of the events that were located in the area close to sensor 1 , due to the large distance of this sensor, the absence of a direct propagation path and the cracking procedure, which did not allow the wave propagation to this direction. Consequently, it is not depicted in the graphs.

As for the wall specimens, the basic conclusions are similar concerning the influence of the propagation distance and heterogeneity. It is important to observe that RT is barely affected by the propagation distance up to sensor 4. However, this index becomes unstable after S4 (distance of $135 \mathrm{~mm}$ from the source) with values at the furthest sensors (S5-S3-S6) between 183 and $231 \mu \mathrm{s}$, while the sensors closer to the origin ranging between 112 and $127 \mu \mathrm{s}$ (S1, S2, S4). As a consequence, the difference increases up to $90 \mu \mathrm{s}$ for a wave propagation of only $15 \mathrm{~mm}$ between sensors 4 and 5 since there is an extra mortar interface. In the case of $\mathrm{AF}$, the trend is decreasing showing again the strongest change between S4 and S5, similar to RT. Initial frequencies of approximately $90 \mathrm{kHz}$ drop down to $52 \mathrm{kHz}$ for sensor $\mathrm{S} 3$ at $154 \mathrm{~mm}$ distance. The accumulation of the imperfections as well as the higher number of distributed cracks in the masonry results in sharp changes along the propagation distance. Consequently, the effect of waveform distortion is more evident, and the frequency is being downgraded.

These interesting outcomes of the experimental investigation on the three different masonry geometries are subsequently compared with the numerical investigation. Figure 8 presents the results of the wave simulation concerning the
AE parameter changes versus the propagation distance in similar fashion as the corresponding experimental results in Fig. 7 for each case of masonry couplet, triplet and wall specimens.

Before the analysis, it is interesting to highlight that due to the ringing effect of the resonant $\mathrm{AE}$ sensors in the experiment, the direct comparison of the absolute values between simulations and experiments is not of importance. Focus is on the changing trends as the propagation distance increases.

In the case of couplets, the numerical trend of RT index (Fig. 8a top) seems to constantly increase till the maximum distance of $100 \mathrm{~mm}$ of sensor 4 following the exactly opposite behavior of AF value. These increasing and decreasing trends in $\mathrm{RT}$ and $\mathrm{AF}$ are in a good agreement with the experimental investigation in couplets, as shown in Fig. 7a. It is observed that RT is highly affected by the propagation distance experimentally, while numerically smaller changes are indicated. The limitations that arise experimentally due to the resonant sensors to receive large differences in the $\mathrm{AF}$ values justify the differences among the experimental and the numerical approach in this limited size geometry of the couplet specimens.

Concerning the triplet specimen, the red line in Fig. $8 \mathrm{~b}$ represents a shear signal excitation, while the black corresponds to tensile excitation. The numerical results follow similar trends compared to the experimental ones showing that RT of signals which are attributed to shear events has higher value in comparison with the tensile ones although with lower differences. Furthermore, the distance effect on
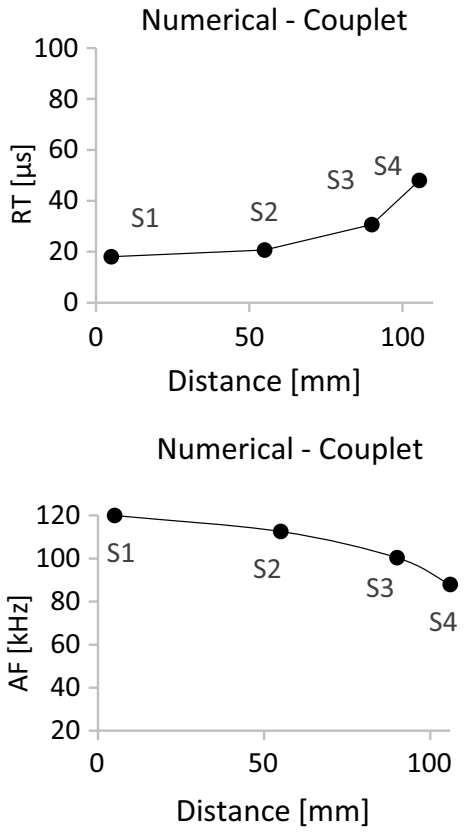

(a)
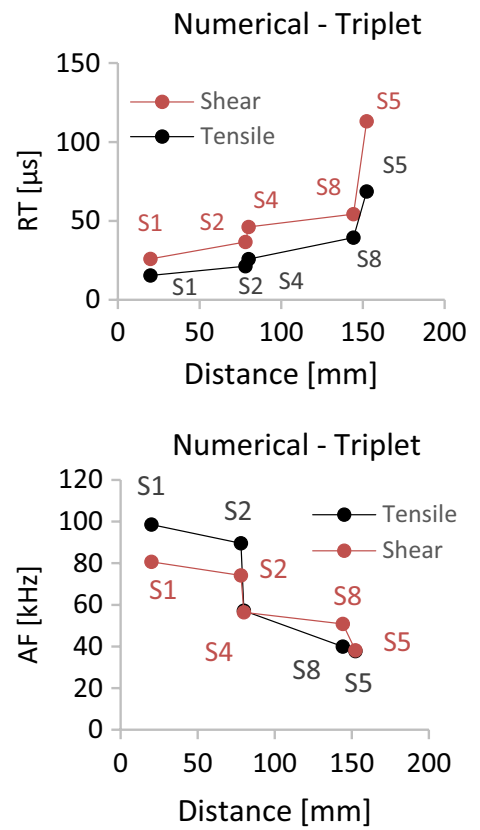

(b)
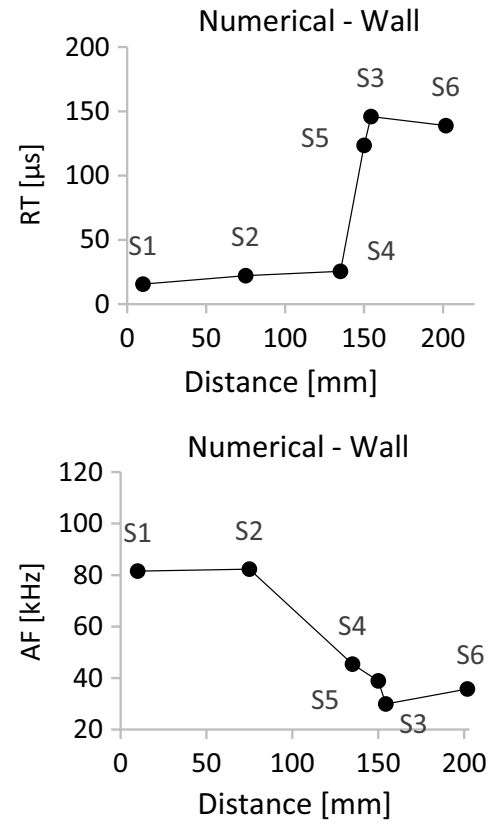

(c)

Fig. 8 Numerical propagation distance effect in RT and AF in a masonry couplets, $\mathbf{b}$ masonry triplets, $\mathbf{c}$ masonry wall specimens 
the propagation is again obvious in both types of excitation. It is noteworthy that the sensor order of increasing values is exactly the same, showing higher rate between S2 and S4 and between $\mathrm{S} 8$ and $\mathrm{S} 5$ both for numerical and experimental results. Finally, the current research has indicated very good agreements in the relative differences of $\mathrm{AE}$ parameters as a function of source-sensor distance and mortar joint crossings. It is the first time that such coupled numerical and experimental approach has been applied in masonry, and the results indicate important issues regarding use of $\mathrm{AE}$ in masonry, which requires further investigations.

Concerning the masonry wall specimen (Fig. 8c), the numerical trend of RT is again in good accordance with the experimental results. In both cases, there is a smooth change in RT for the first three sensors, while this becomes more sharp between sensors 4 and 5 where the propagated distance is only $15 \mathrm{~mm}$. Consequently, the more remotely the signal propagates, the more increasing effect there will be in the value of RT showing that there is a strong dependence of RT evolution with the propagation distance. However, it is characteristic that for the most remote sensor (S6), both experiments and simulations show a decrease in RT which could be the result of the increased attenuation which diminishes the peak amplitudes. Concerning the AF, it is interesting to mention the similar reduction tendency between the experimental and the numerical approaches for the same type of tests (compression) on the couplet and wall specimens. Experimentally, AF presents a decrement of $18.5 \mathrm{kHz}$

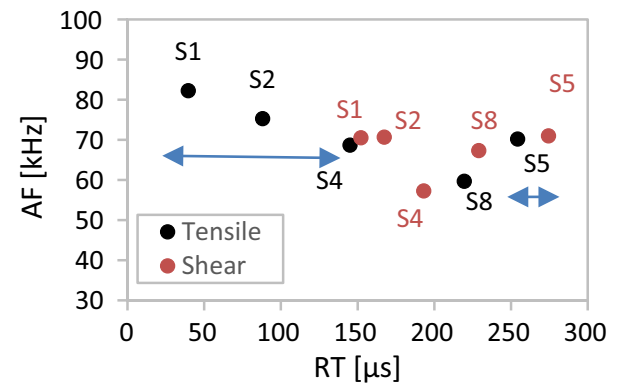

Fig. 9 Effect of experimental propagation distance in $\mathrm{RT}$ and $\mathrm{AF}$ in the couplet specimens in a distance of $85 \mathrm{~mm}$, while it reaches difference decrease of $36.1 \mathrm{kHz}$ in a distance of $144 \mathrm{~mm}$ in the wall specimen due to attenuation and reflections in the interfaces. This comes in agreement with the numerical investigation where AF decreases approximately $20 \mathrm{kHz}$ in the couplet and much more in the wall specimen, as shown in Fig. 8 for the same distances.

Figure 9 shows a summarized comparison of the results presented in Fig. $7 b$, concerning the experimental and numerical approach of the triplet specimen. In Fig. 9, we can clearly observe how tensile and shear indices received by the closest sensor S1 and the remote ones are shifted to higher $\mathrm{RT}$ and lower AF values due to the effect of the propagation distance. For instance, the RT in the case of a tensile crack is $7-8$ times higher at $\mathrm{S} 5$ (most remote $-150 \mathrm{~mm}$ ) than $\mathrm{S} 1$ (20 mm-closest). Also, the difference between the RT among the different excitations (tensile-shear) is large for $\mathrm{S} 1$ (indicated by the long blue arrow) but much smaller for the most remote sensor S5 (short arrow).

It is also interesting to highlight in Fig. 10 the difference in the received numerical waveforms from the closest $\mathrm{S} 1$ and the most remote $\mathrm{S} 5$ sensor for the same tensile excitation. It illustrates how the waveform shape changes according to the distance which results in $\mathrm{AE}$ feature differences as well. This specific example concerns a tensile excitation, but similar behavior was followed also in the case of a shear excitation pulse.

Finally, in all of the aforementioned cases, the increasing and decreasing trend in RT and AF, respectively, is indicative of the strong dependence of the AE parameters on the propagation distance to the sensors. The similarity of the experimental and numerical trends renders this investigation encouraging for validation and prediction of the changes in the $\mathrm{AE}$ parameters.

\section{Conclusions}

The present article deals with the dependence of AE parameters on the propagation distance. This is one of the first times to the authors' knowledge that the effect of wave
Fig. 10 Effect of propagation distance in RT and AF numerically-tensile excitation waveforms in triplet received from a closest $\mathrm{S} 1$, b most remote $\mathrm{S} 5$ sensors

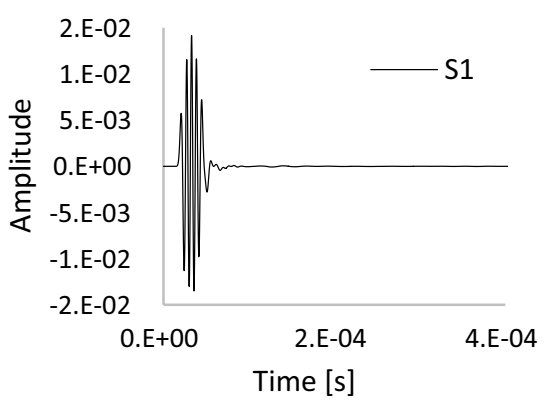

(a)

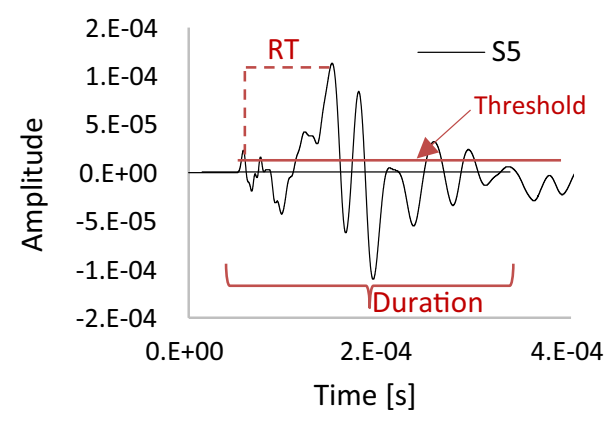

(b) 
propagation is investigated in masonry components for $\mathrm{AE}$ study. Masonry is a highly heterogeneous material, and this makes this need imperative. AE has shown the capacity of characterizing the fracture mode in individual constituents (bricks and mortar). However, when upscaling the application to masonry components, it was shown that crucial indices such as the AF and the RT, which can be used for fracture mode determination, are strongly influenced by the propagation distance in all of the three geometries that were investigated, masking therefore the source information contained in the initial pulse. Therefore, it is obvious that the effect of attenuation has strong influence on the AE parameters value. This should be taken into account before applying crack classification approaches in masonry components and structures. Practically, concerning the wall geometry, it seems that $\mathrm{AE}$ parameters are close to the original values for distances within $150 \mathrm{~mm}$ from the source, while afterward, they become unstable. Secondly, it was shown that experimental trends of RT and AF of small and larger masonry specimens are in good agreement with the numerical simulations. This is encouraging as it enables the numerical validation of experimental $\mathrm{AE}$ analysis and possible prediction of expected AE signal characteristics.

As a future step, sensors with lower frequency response could be used to have less damping, improving the characterization power of frequency indicators. For small distances, it seems that a single parameter (RT) is indicative of the fracture mode. However, when expanding to larger distances, other parameters that pose also some characterization power, like AF, can also be used to enhance the accuracy of characterization. In addition, more experimental validations and further investigation concerning the effort to deduce $\mathrm{AE}$ parameters taking out the signal distortion due to the propagation distance effect would be valuable in order to make a robust tool for an accurate prediction of the AE scattering in masonry materials.

Acknowledgements The Research Fund-Flanders (FWO) is acknowledged for funding the FWO project "AE-FracMasS: advanced Acoustic Emission analysis for Fracture mode identification in Masonry Structures" (G.0C38.15)

\section{References}

1. Carpinteri A, Lacidogna G, Pugno N. Structural damage diagnosis and life-time assessment by acoustic emission monitoring. Eng Fract Mech. 2007;74(1-2):273-89.

2. Wevers M. Listening to the sound of materials: Acoustic emission for the analysis of material behaviour. NDT E Int. 1997;30(2):99106. https://doi.org/10.1016/S0963-8695(96)00051-5.

3. Verstrynge E, Schueremans L, Van Gemert D, Wevers M. Monitoring and predicting masonry's creep failure with the acoustic emission technique. NDT E Int. 2009;42(6):518-23. https://doi. org/10.1016/j.ndteint.2009.03.001.
4. Kravchuk R, Landis EN. Acoustic emission-based classification of energy dissipation mechanisms during fracture of fiber-reinforced ultra-high-performance concrete. Constr Build Mater. 2018;176:531-8. https://doi.org/10.1016/j.conbuildma t.2018.05.039.

5. Shiotani T, Ohtsu M, Ikeda K. Detection and evaluation of AE waves due to rock deformation. Constr Build Mater. 2001;15(56):235-46. https://doi.org/10.1016/S0950-0618(00)00073-8.

6. Recommendation of RILEM TC 212-ACD: acoustic emission and related NDE techniques for crack detection and damage evaluation in concrete. Test method for classification of active cracks in concrete structures by acoustic emission RILEM Technical Committee. Mater Struct. 2010;43(9):1187-9. https://doi.org/10.1617/ s11527-010-9640-6.

7. Goszczyńska B, Świt G, Trąmpczyński W, Krampikowska A, Tworzewska J, Tworzewski P. Experimental validation of concrete crack identification and location with acoustic emission method. Arch Civ Mech Eng. 2012;12(1):23-8. https://doi.org/10.1016/j. acme.2012.03.004.

8. Ohno K, Ohtsu M. Crack classification in concrete based on acoustic emission. Constr Build Mater. 2010;24(12):2339-46. https://doi.org/10.1016/j.conbuildmat.2010.05.004.

9. Farhidzadeh A, Salamone S, Singla P. A probabilistic approach for damage identification and crack mode classification in reinforced concrete structures. J Intell Mater Syst Struct. 2013;24(14):1722-35.

10. Kourkoulis SK, Dakanali I. Pre-failure indicators detected by acoustic emission: Alfas stone, cement-mortar and cement-paste specimens under 3-point bending. Frattura ed Integrità Strutturale. 2017;11(40):74-84.

11. Mpalaskas AC, Matikas TE, Van Hemelrijck D, Papakitsos GS, Aggelis DG. Acoustic emission monitoring of granite under bending and shear loading. Arch Civ Mech Eng. 2016;16(3):313-24. https://doi.org/10.1016/j.acme.2016.01.006.

12. Livitsanos G, Shetty N, Hündgen D, Verstrynge E, Wevers M, Van Hemelrijck D, Aggelis DG. Acoustic emission characteristics of fracture modes in masonry materials. Constr Build Mater. 2018;162:914-22. https://doi.org/10.1016/j.conbuildma t.2018.01.066.

13. Aggelis DG, Shiotani T, Papacharalampopoulos A, Polyzos D. The influence of propagation path on elastic waves as measured by acoustic emission parameters. Struct Health Monit. 2012;11(3):359-66.

14. Ohtsu M. Innovative AE and NDT techniques for on-site measurement of concrete and masonry structures. RILEM State Art Rep. 2016;20:89-103.

15. Aggelis DG, Matikas TE. Effect of plate wave dispersion on the acoustic emission parameters in metals. Comput Struct. 2012;98 99:17-22. https://doi.org/10.1016/j.compstruc.2012.01.014.

16. Ohtsu M, Ono K. The generalized theory and source representations of acoustic emission. J Acoust Emiss. 1986;5(4):124-33.

17. Rhian Green E. Acoustic emission in composite laminates. J Nondestruct Eval. 1998;17(3):117-27. https://doi.org/10.1007/BF024 46115.

18. Sause MGR, Horn S. Simulation of acoustic emission in planar carbon fiber reinforced plastic specimens. J Nondestr Eval. 2010;29(2):123-42. https://doi.org/10.1007/s10921-010-0071-7.

19. Brigante M. Calculation of wave structure of the ultrasonic beams in nondestructive testing of brick masonries. Mech Res Commun. 2014;59:58-63. https://doi.org/10.1016/j.mechrescom .2014.04.005

20. De Santis S, Tomor AK. Laboratory and field studies on the use of acoustic emission for masonry bridges. NDT E Int. 2013;55:64 74. https://doi.org/10.1016/j.ndteint.2013.01.006.

21. Livitsanos G, Shetty N, Verstrynge E, Wevers M, Van Hemelrijck D, Aggelis DG. Characterization of fracture mode in historical 
masonry mortars by acoustic emission. In: 2nd International RILEM/COST conference on early age cracking and serviceability in cement-based materials and structures; 2017. p. 125-30.

22. Livitsanos G, Shetty N, Verstrynge E, Wevers M, Van Hemelrijck D, Aggelis DG. NDT fracture mode characterization in masonry components made with different mortars. In: Proceedings of the structural faults and repair 2018; 2018. https://doi.org/10.25084/ sfr.2018.0031.

23. Singh S, Munjal P. Bond strength and compressive stress-strain characteristics of brick masonry. J Build Eng. 2017;9:10-6. https ://doi.org/10.1016/j.jobe.2016.11.006.

24. Alecci V, Fagone M, Rotunno T, De Stefano M. Shear strength of brick masonry walls assembled with different types of mortar. Constr Build Mater. 2013;40:1038-45. https://doi.org/10.1016/j. conbuildmat.2012.11.107.

25. Mojsilović N, Simundic G, Page AW. Static-cyclic shear tests on masonry triplets with a damp-proof course membrane. In: Proceedings of the 12 th Canadian masonry symposium. Vancouver; 2013.

26. Hendrickx R. The adequate measurement of the workability of masonry mortar, PhD Thesis, KU Leuven, Civil Engineering Department; 2009.
27. Mistras Group Inc. "AEwin Software", products \& systems division. NY: Princeton Junction; 2009.

28. Shiotani T. Parameter analysis. In: Grosse C, Ohtsu M, editors. Acoustic emission testing. Berlin: Springer; 2008. p. 41-51. https ://doi.org/10.1007/978-3-540-69972-9_4.

29. Wave2000. NY: Cyber-Logic, Inc. http://www.cyberlogic.org.

30. Moser F, Jacobs LJ, Qu J. Modeling elastic wave propagation in waveguides with the finite element method. NDT E Int. 1999;32(4):225-34.

31. Shetty N, Livitsanos G, Aggelis DG, Van Hemelrijck D, Wevers $M$, Verstrynge E. Acoustic emission characterization of fracture modes in masonry under direct shear test. In: 24th International acoustic emission symposium (IAES), 5-9 November 2018, Sapporo, Japan; 2018.

Publisher's Note Springer Nature remains neutral with regard to jurisdictional claims in published maps and institutional affiliations. 